\title{
Fingerprint information database system based on image processing technology
}

\author{
Zhao Cai, Miao Xiang Lin, Xu Dawei \\ Xi'an Jiaotong University City College, Xi'an 710018,China
}

Key words: information age; social security management; fingerprint database; image processing; fingerprint comparison

Abstract. In the information age, with the continuous development of science and technology, the computer plays an increasingly important role in the public security system of social security management. For the investigation, the emergence of computer automatic fingerprint identification system has changed the traditional method for fingerprint matching, improve the efficiency and quality of fingerprint query, quickly and accurately is cracked to provide the scientific basis for the determination of the direction of the investigation provide clues. This time, in order to optimize the fingerprint database for example, using the image processing method of fingerprint quality were optimized, and the comparison results were tested, verified the important role of information on social security management system, which provides a more meaningful reference for the research of the modern social security management.

\section{Introduction}

With the continuous development of computer technology, especially the emergence of cloud computing platform, the current society has begun to enter the era of information, provides great convenience for the development of computer management of social security and public security system, including information of fingerprint identification system is a typical. Fingerprint recognition system is a typical pattern recognition system, which includes fingerprint image acquisition, processing, feature extraction and specific peer to peer module. The fingerprint image acquired by scanner, digital camera etc. there is a certain ambiguity, fingerprint texture clarity can not meet the requirements of the system, and the use of computer image processing technique for fingerprint image optimization, so as to meet the requirements of the system, has an important significance for the establishment of fingerprint information database.

\section{The establishment of public security fingerprint database for public security management}

In the information age, the establishment of public security fingerprint database has important significance for the management of social security, establish the fingerprint database of accurate and reliable to need to expand the scope of collection, and using ten fingers all the fingerprint data acquisition methods, and therefore will have a massive, and refers to the quality of the data lines is directly related to the fingerprint matching speed and the accuracy rate of. At present, the acquisition of fingerprint is usually done by pressing and collecting images. Besides the need to improve the acquisition accuracy of the device, it is also necessary to combine certain image processing technology. The optimization process of this fingerprint database system is as follows. 


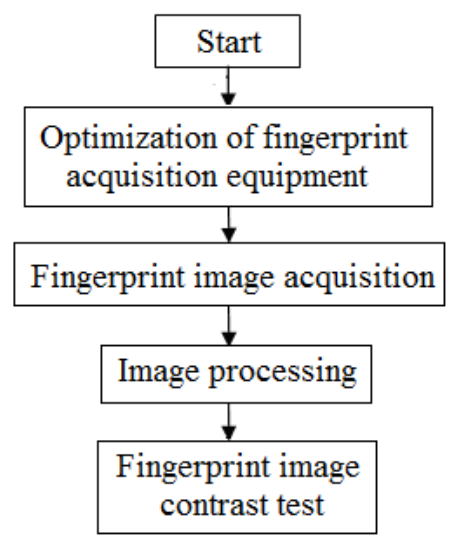

Fig.1 The optimization process of fingerprint database system

As shown in Figure 1, the optimization of fingerprint database system can not only improve the technical index of fingerprint acquisition device, but also improve the quality of fingerprint image through the improvement of image processing method, so as to improve the accuracy of fingerprint matching. The further optimization of the system can also be used to verify the reliability of the system by the fingerprint contrast test, thus providing the technical index for the next step optimization.

\section{The enhancement and optimization of fingerprint information based on image processing technology}

In order to make the texture of fingerprint image more clear, first, we can transform the color fingerprint image into gray image by grayscale processing, the number of gray image is generally 8 bits, and the number of color image is generally 24 bits, and the conversion formula is as following:

$$
C=x R+y G+z B, x+y+z=1
$$

In which, $R, G, B$ represent the three basic colors, while the coefficients $x, y, z$ represen the 3 basic colors, $C$ indicates the color obtained after the grayscale processing.

In order to make the fingerprint recognition system image with uniform size, for fingerprint alignment, need the image is normalized, normalized to the nearest neighbor interpolation, the zoom of the image processing, to achieve the unified standard zoom ratio $f_{x}$ hypothesis in the $X$ axial direction is in the $Y$ axial direction is the ratio $f_{y}$ of the original image $f(x, y)$ to zoom. Among them, a point $\left(x_{0}, y_{0}\right)$ corresponding to the image $g(x, y)$ zoom after point $\left(x_{1}, y_{1}\right)$, the calculation expressions is as following:

$$
\begin{array}{r}
x_{1}=f_{x} \times x_{0} \\
y_{1}=f_{y} \times y_{0}
\end{array}
$$

After the gray normalization is used, the gray value of the image can be distributed uniformly in the range of $[0,255]$. The calculation formula for the gray normalization of the gray scale can be calculated. 


$$
p(i, j)=\frac{p^{\prime}(i, j)-G_{1}}{G_{2}-G_{1}} \times 255
$$

The fingerprint image normalization, fingerprint can be further enhanced, enhanced processing can make the fingerprint texture more clearly and make the fingerprint black texture is more obvious, the valley position more white, will connect the wire, fingerprint image enhancement can be achieved through fingerprint smoothing and filtering operation, which is to cut $V_{\omega}=\frac{1}{7}(-3,-1,3,9,3,-1,-3)$, the filter template $H_{\omega}=\frac{1}{7}(1,1,1,1,1,1,1)$. Method to filter template is enhanced by image intensity normalization and the effect of fingerprint image, as shown in Figure 2 .
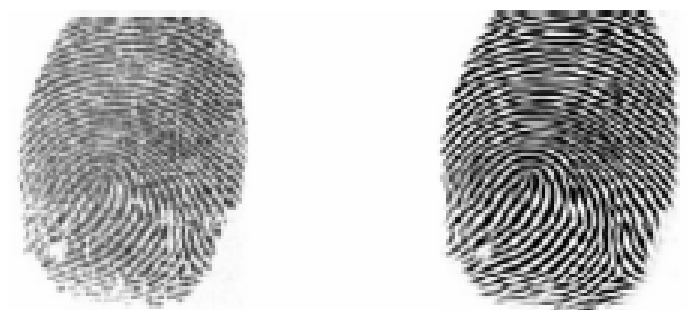

(a) fingerprint original image (b) gray normalized enhancement image

Fig.2 Gray normalized image enhancement before and after enhancement

In order to improve the accuracy of fingerprint matching, we can further refine the fingerprint image. The thinning can make the texture more obvious and improve the efficiency of fingerprint identification.
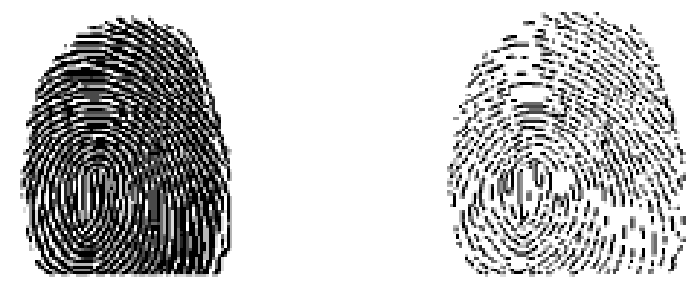

(a) the pre thinning image (b) after thinning the image

Fig.3 Comparison between pre and post thinning images

As shown in Figure 3, the texture of the fingerprint image is more obvious after the thinning of the fingerprint, thus effectively improving the efficiency of the fingerprint alignment.

\section{The recognition and matching system test of fingerprint image information}

Fingerprint recognition system is a typical pattern recognition system, which includes fingerprint image acquisition, processing, feature extraction and specific peer to peer module. After collecting the image through the hardware, we need to compress and deal with the image further. Finally, the matching is optimized.

In order to compare the image, it can be realized with the Hu's invariant moments in the discrete state of the image, and the ordinary moment of the image order can be written.

$$
m_{p q}=\sum_{m=1}^{M} \sum_{n=1}^{N} m^{p} n^{q} f(m, n)
$$

The calculation formula for the image center distance of $p+q$ rank is as following: 


$$
u_{p q}=\sum_{m=1}^{M} \sum_{n=1}^{N}(m-\bar{x})^{p}(n-\bar{y})^{q} f(m, n)
$$

The normalized central moments are used to transform the image into matrix variables with translation, scale change and rotation invariance. Finally, the image is compared, and the matching results shown in Figure 4 are obtained through this comparison.

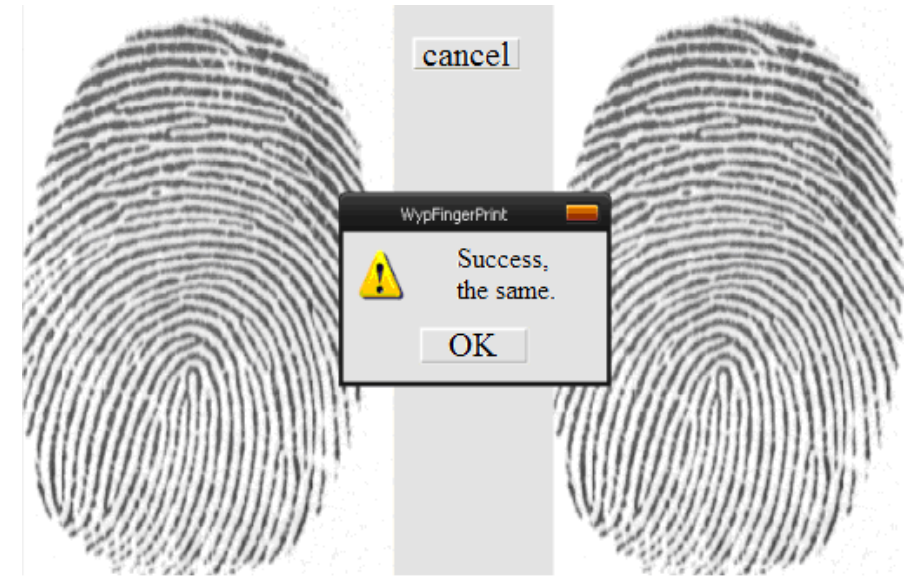

Fig. 4 The authentication results of information system of fingerprint identification

As shown in Figure 4 represents the results of fingerprint identification information system, with a fingerprint two collected in comparison in the system, the results can be seen that the system can successfully recognize a person's fingerprint, so as to verify the reliability of the system.

\section{Conclusion}

The advent of the information age has brought great convenience to the design of social security management and public security information system, the information of the fingerprint recognition system of fingerprint image processing and recognition are studied, and the development of related recognition platform to validate the algorithm. In the future research, we can also develop a more powerful system with cloud platform to further develop fingerprint classification, compression and database parts, so as to form a complete set of social security management and public security information fingerprint recognition system.

\section{Reference}

[1] He Yao, Wang Wenqing, Sheffi. Research on mass data mining based on cloud computing [J]. Computer technology and development, Vol.23 (2) , (2013), p. 69-72

[2] Ding Yan, Yang Qingping, Qian Yuming. Platform architecture and its key technology research of [J]. ZTE cloud based data mining technology, Vol.19 (1), (2013), p.53-60

[3] Li Kai, Chang Zheng. Design and implement based on cloud computing parallel data mining system[J]. Microcomputer information, Vol.27 (6) , (2011), p.121-123

[4] Dan Yong. Data governance and innovation of social security and prevention and control system [J]. Studies on Socialism with Chinese Characteristics. Vol.38(4), (2015)

[5] Shi Liping, Zhang Yinghui, Luo Aling. The big data industry development opportunities and [J]. Software guide. No.07, (2015)

[6] He Xiaoping, Huang long. The evolution path of the large data field, research hot spots and visual analysis of frontiers [J]. Journal of Modern Information. No.04, (2015)

[7] Shiping. Innovative government social management [J]. Oriental Enterprise Culture in the big data age. Vol.19, (2014)

[8] Zhang Zhaoduan. Strategic thinking on the construction of public security data [J]. Journal of 
Chinese People's Public Security University (Social Science Edition). No.04, (2014)

[9] Fan Xiaoqian, Li Xinzhi. Public security prevention and control in the era of big data - key exploration of data processing countermeasures [J]. Theorists. No.08, (2014)

[10] Ma Jianguang, Jiang Wei. The big data concept, characteristics and application of [J]. Defense technology. No.02, (2013)

[11] sun Guang Lu, Qi Haoliang. Journal of Tsinghua University [J]. online scheduling of spam filtering based on logistic regression, Vol.53 (5), (2013), p.734-740

[12] Liu Botao. Research on data mining algorithms based on rough sets [J]. technology in Western China, Vol.10 (14), (2011), p.11-12

[13] Shi Junru, Heimin star, Yang Jun. A IOT data management framework of [J]. computer science, Vol.10 (12), (2015), p.294-298.

[14] Li Xinlong, Zhang Ling, Li Kun, etc., and so on. Data allocation strategy for sensor information of the Internet of things, [J]. computer research and development, Vol.10 (15), (2013), p.297-305.

[15] Zhang Yan. Analysis and research of database technology based on the mass data processing of the Internet of things [J]. Computer optical disc software and applications, Vol.2 (19), (2014), p.97-99

[16] Yao Lin. Police strategy in the era of big data [J]. Journal of Beijing Police Academy. No.04, (2014). 